\title{
Comparison of Nonoriented and Grain-Oriented Material in an Axial Flux Permanent-Magnet Machine
}

\author{
Damian Kowal ${ }^{1}$, Peter Sergeant ${ }^{1,2}$, Luc Dupré ${ }^{1}$, and Alex Van den Bossche ${ }^{1}$ \\ ${ }^{1}$ Department of Electrical Energy, Systems and Automation, Faculty of Engineering, Ghent University, B-9000 Ghent, Belgium \\ ${ }^{2}$ Department of Electrotechnology, Faculty of Applied Engineering Sciences, University College Ghent, B-9000 Ghent, Belgium
}

\begin{abstract}
The performance and iron losses of an axial flux permanent-magnet synchronous machine (AFPMSM) using nonoriented (NO) steel are compared with the performance and iron losses of an AFPMSM using grain-oriented (GO) material. The machine is modeled by several 2-D finite element models in circumferential direction, at different radii. The material model for the GO material is an anhysteretic anisotropic model based on the magnetic energy. The magnetic energy is computed by using several measured quasi-static $B H$-loops on an Epstein frame in seven directions starting from the rolling direction to the transverse direction. The losses are calculated $a$ posteriori, based on the principles of loss separation and dynamic loop measurements. A loss model was made for each of the seven directions, assuming unidirectional fields. In comparison with the more usual NO material, both the saturation induction and the torque are higher with GO material. The magnetic field in the GO material is lower than for NO material in the major part of the iron, but higher in the tooth tips where the field is not in the rolling direction. The stator iron losses are about 7 times lower for the considered GO compared to the NO material.
\end{abstract}

Index Terms_Finite element methods, losses, permanent magnet machines.

\section{INTRODUCTION}

A XIAL flux permanent-magnet synchronous machines (AFPMSMs) have become popular in the last few decades because they may have a higher torque at low speed in comparison with radial flux machines. They can be attractive when the axial length is small and the pole number is high [1].

The property of high torque at low speed is interesting for several applications, such as traction and energy generation. The AFPMSM is investigated as a high efficiency generator for e.g., wind energy systems, in particular "direct-drive" generators with direct coupling to the shaft, causing the nominal speed of the machine to be very low. In [2] and [3], a single stator AFPMSM, respectively a dual stator AFPMSM is designed for direct-drive wind applications.

In literature, many aspects have been investigated. A first aspect is the type of windings. AFPMSMs - but also radial PMSMs - can have either distributed windings on the stator, or concentrated pole windings. The advantage of concentrated pole windings is the easy manufacturing, the short coil ends, and the higher iron section for a given coil dimension. A disadvantage is that for a three-phase machine several windings should be connected in series. When connecting $n$ concentrated windings in series, the total electromotive force is not $n$ times the voltage of a single winding, because of phase shift in neighboring windings. An alternative is to make a machine with many phases [3].

Cogging torque reduction is another aspect that has been studied intensively in literature. This can be done by the skewing of magnets and the displacement of stator teeth [4]. Another possibility is to use a coreless machine [2].

Manuscript received June 20, 2009; revised August 28, 2009; accepted August 31, 2009. Current version published January 20, 2010. Corresponding author: P. Sergeant (e-mail: Peter.Sergeant@UGent.be).

Color versions of one or more of the figures in this paper are available online at http://ieeexplore.ieee.org.

Digital Object Identifier 10.1109/TMAG.2009.2032145
The aspect of the material choice in the machines with stator core is not much investigated. Most electrical machines use laminated nonoriented (NO) silicon steel, but also grain-oriented (GO) material is possible in some cases. For AFPMSM, the stator is difficult to make by stacking thin laminations regardless if they are NO or GO. In [4], the stator core and stator teeth are manufactured from a single silicon iron strip that is wound in a spiral construction. Based on the given $B H$-characteristic, it can be assumed that this machine is made in grain oriented material, although the authors do not mention it. As the flux in the stator teeth is perpendicular to the flux in the stator core, it is impossible to benefit from the GO material in both the stator core and the stator teeth.

An alternative to thin sheets of silicon steel is the use of soft magnetic composites (powdered iron) [5]. Manufacturing 3-D shapes is possible with this material. The disadvantage of this material is the relatively low permeability, and higher losses at low frequencies. Because of the high resistivity, the cited paper shows that the losses of iron powder (Somaloy 550+0.6\%LB1) become lower than those of NO Si steel (Cogent M400-50A) for a frequency higher than about $1 \mathrm{kHz}$.

The AFPMSM studied in this paper is designed for a speed of $2000 \mathrm{rpm}$ and a power of about $3.6 \mathrm{~kW}$, for use in a combined heat and power application. As the machine has 16 poles, the nominal stator frequency is quite high: $267 \mathrm{~Hz}$. At this frequency, it is necessary to choose a stator with sufficiently thin sheets in order to limit the iron losses. The chosen machine configuration was the same as [6]: a single stator, dual rotor configuration with concentrated stator coils wound around stator teeth in laminated soft magnetic material.

The machine uses GO instead of NO soft magnetic material, with an appropriate stacking method explained in the next section. Due to the unidirectional magnetic flux in the majority of the stator volume, it is expected to have advantages in performances and efficiency of AFPMSM by using GO material in the stator laminations. These advantages are clarified in the following sections by a detailed comparison of a AFPMSM with GO and with NO material, concerning electromotive force 


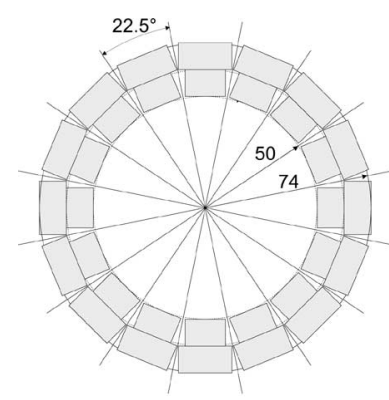

(a)

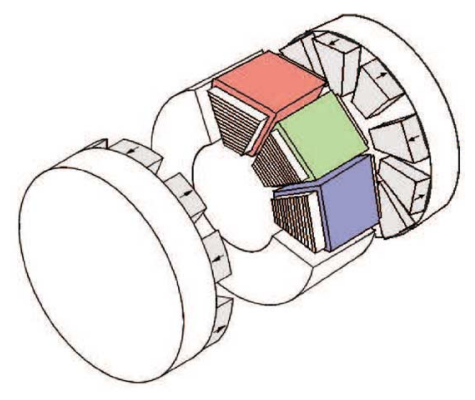

(b)
Fig. 1. (a) Rotor geometry of the AFPMSM; dimensions in mm and (b) single stator dual rotor configuration.

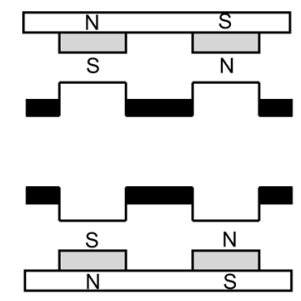

(a)

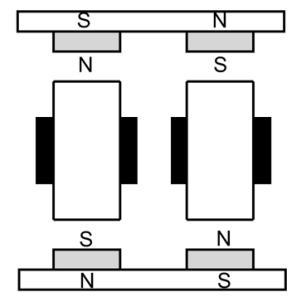

(b)
Fig. 2. Single stator dual rotor AFPMSM with (a) axial (vertical) field in the stator teeth and azimuthal (horizontal) field in the stator yoke; and (b) axial field in the stator teeth.

(EMF), torque and losses. The chosen GO material is a relatively good quality material $(0.3 \mathrm{~mm}$ thickness, $1 \mathrm{~W} / \mathrm{kg}$ in the rolling direction at $50 \mathrm{~Hz}$ and $1.5 \mathrm{~T}$ ), while the $\mathrm{NO}$ material is a rather low quality material $(0.5 \mathrm{~mm}$ thickness, $7 \mathrm{~W} / \mathrm{kg}$ at $50 \mathrm{~Hz}$ and $1.5 \mathrm{~T}$ ). By comparing two materials that are strongly different, it can be observed if it is worth the extra cost of choosing high quality material for an AFPMSM.

\section{Axial Flux Permanent-Magnet Machine}

The AFPMSM with single stator and dual rotor is shown in Fig. 1. Two possibilities exist for this machine with single stator and dual rotor. In the first possibility, the facing magnets on both rotors have opposite magnetization orientation, and the stator has both a yoke and teeth [Fig. 2(a)]. The second possibility has facing magnets with equal magnetization orientation, and a stator that consists of teeth only [Fig. 2(b)]. The second type was preferred for two reasons: 1) the absence of a yoke reduces the weight of iron, and by consequence reduces the iron losses and cost; 2) the magnetic field is expected to be along the axial direction in the majority of the yoke, which may justify the choice of GO material.

\section{A. Geometry and Materials}

To the surface of each of the two rotor discs, 16 T-shaped, axially-polarized magnets are glued as presented in Fig. 1. The T-shape magnets from Vacuumschmelze have a remanence of $1.26 \mathrm{~T}$ and they consist of small segments in order to reduce eddy current losses. Both rotor yokes were made of solid construction steel of $8 \mathrm{~mm}$ thickness. The shaft is made from aluminum.

In the stator, the 15 teeth are made of $0.3 \mathrm{~mm}$ thick laser-cut GO laminations of $1.0 \mathrm{~W} / \mathrm{kg}$ losses at $1.5 \mathrm{~T}$ and $50 \mathrm{~Hz}$. The

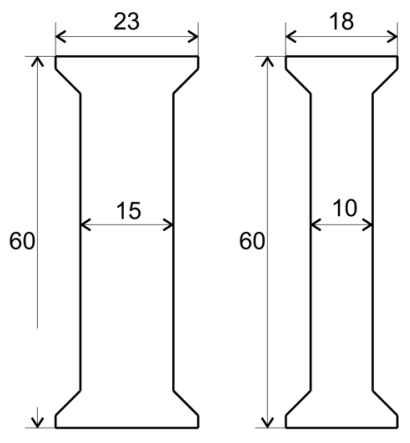

(a)

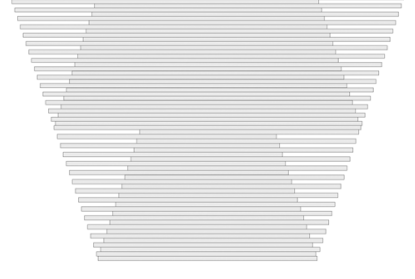

(b)
Fig. 3. (a) Stator laminations [mm] and (b) Cross-section of stacked laminations in stator tooth.

nonoriented material used in the simulations is a M700-50A with $0.5 \mathrm{~mm}$ thickness of the sheets. The following technique was used to make the 3-D shape for the stator. Two different geometries of I-profiles were laser-cut [Fig. 3(a)] and stacked with overlap zone: as depicted on Fig. 3(b), the bottom part is made of a narrower lamination profile while the part on the top is wider. This method makes it possible to make a 3-D geometry with only two shapes of profiles and a slight reduction of the fill factor: in the (small) overlap zones, the fill factor is only $50 \%$. A concentrated copper winding is put around each stator tooth in two layers with 90 turns in total, resulting in a simple and robust winding.

\section{B. Numerical Model}

An axial flux PMSM has intrinsically a 3-D geometry. As the conventional 2-D FEM to study a machine is not possible for this type of machine, many alternatives have been proposed in literature. In [7], analytical methods were developed to compute the no-load and armature fields in the AF generator. The analytical method assumes an infinite permeability of the rotor back iron. The stator is coreless. The analytical method is fast to evaluate, but it does not take into account magnetic saturation, so that superposition of no-load and armature field is allowed. Also in [8], an analytical technique is used: the vector potential equation is solved by separation of variables. Also here, several "simplifying" assumptions had to be made, so that the method becomes "quasi 3-D" rather than 3-D.

In order to avoid a computationally intensive numerical model, the machine is modeled by several layers of 2-D models with geometry and boundary conditions in Fig. 4. Only 5 magnets and 5 teeth (1/3) of the machine are modeled. The 2-D models are taken in azimuthal direction, along the circumference of the machine, like in [9]. In total, six layers were chosen with unequal thicknesses, as shown in Fig. 5. As shown in the geometry of Fig. 1, the magnets have a T-shape: they are small at small radius (layers 1-3) and wider at large radius (layers 4-6). This corresponds to the stator laminations: at layers 1-3, the small I-cores of Fig. 3(a) are taken, while at layers 4-6, the wide I-cores are modeled. Hence, in each layer, the overlap region is different.

Concerning the electromagnetic properties, the rotor iron is modelled by a constant permeability of $2000 \mu_{0}$, because the thickness of the rotor iron is sufficient to avoid saturation. The 


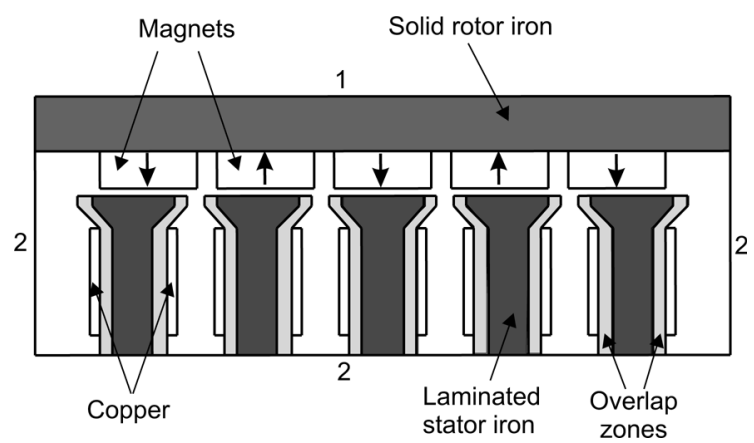

Fig. 4. Modeled geometry of the AFPMSM and boundary conditions: 1) is Dirichlet and 2) is Neumann.

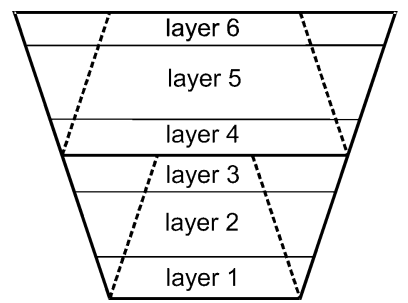

Fig. 5. Division of the geometry in layers for the 2-D FEM, illustrated on a stator tooth. The dotted lines are the edges of the overlap zones.

magnets are represented by their recoil line with slope $\mu_{0}$. For the stator iron, a different approach is chosen for NO and GO material. For NO material, a nonlinear characteristic $\mu(B)$ is determined. The equation for the vector potential $\mathbf{A}$ is

$$
\nabla \times\left(\frac{1}{\mu_{0} \mu_{\mathrm{r}}} \nabla \times \mathbf{A}\right)=\mathbf{J}_{\mathrm{e}}
$$

with $\mathbf{J}_{\mathrm{e}}$ the external current density, $\mu_{0}$ the permeability of vacuum and $\mu_{\mathrm{r}}$ the relative permeability. For the GO material, an anisotropic material model was made that gives the magnetization $\mathbf{M}(\mathbf{B})$. The equation is then

$$
\nabla \times\left(\frac{1}{\mu_{0}} \nabla \times \mathbf{A}\right)-\nabla \times \mathbf{M}=\mathbf{J}_{\mathrm{e}}
$$

The waveforms of the magnetic induction are recorded in several points of the geometry, for a posteriori calculation of losses in the magnetic material. In regions with changing direction of magnetic flux, a dominant direction was recognized and applied for loss evaluation.

\section{MATERIAL MODELS}

\section{A. Epstein Measurements}

From the considered GO material, several Epstein strips were cut from a sheet of GO material in seven different directions. The rolling direction (RD) - along the $x$-axis-is chosen as a reference, and is assigned angle $0^{\circ}$. The other directions have angles of $15^{\circ}, 30^{\circ}, 45^{\circ}, 60^{\circ}, 75^{\circ}$, and $90^{\circ}$, which is the transverse direction (TD), as shown in Fig. 6. We preferred Epstein measurements instead of single sheet measurements, see [10], because the dimensions of the Epstein strips approximate to a large extent the dimensions of the I-profiles of the machine (Fig. 3).

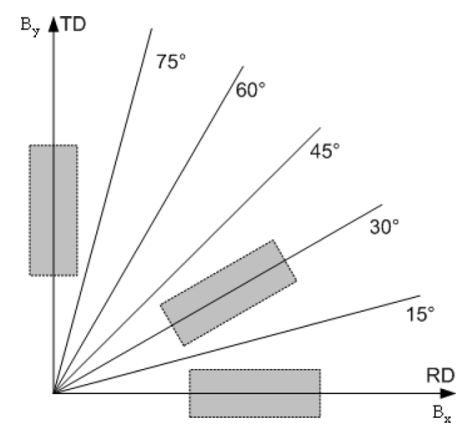

Fig. 6. Directions in $\left(B_{x}, B_{y}\right)$ plane along which the samples were cut.

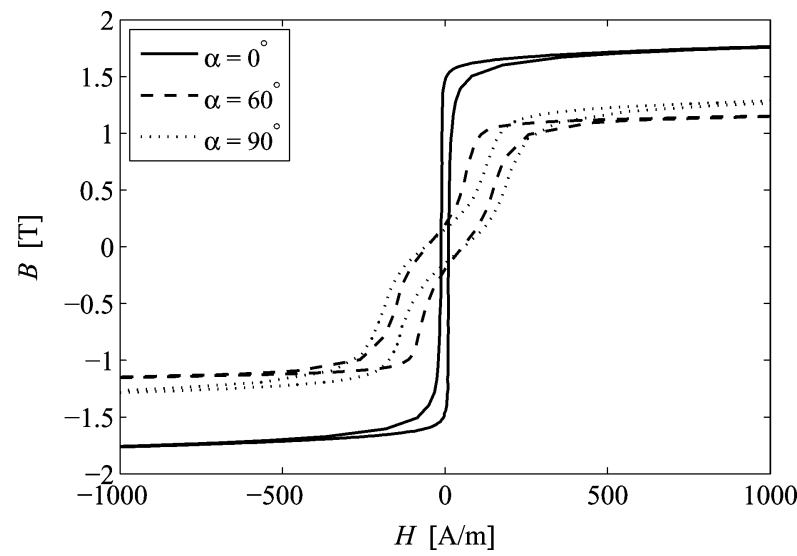

Fig. 7. (a) Hysteresis loops for $7200 \mathrm{~A} / \mathrm{m}$ for $\mathrm{GO}$ material along the $0^{\circ}$ (RD), $90^{\circ}$ (TD), and the $60^{\circ}$ direction.

For all GO strips, several quasi-static $B H$-loops up to 7200 $\mathrm{A} / \mathrm{m}$ were measured on an Epstein frame, resulting in $B(H)$ characteristics along all directions. These hysteresis loops are used for the energy based material model (Section III-B) used in the finite element routines. Several dynamic loops were measured up to $200 \mathrm{~Hz}$ frequency for the loss model explained in Section III-C. Evidently, the peak induction $B_{\mathrm{p}}$ in the RD was much higher than in the other directions, see Fig. 7.

For the NO material, quasi-static and dynamic hysteresis loops were measured on a ring core, for $H$ fields up to 4000 $\mathrm{A} / \mathrm{m}$ and $200 \mathrm{~Hz}$ (Fig. 8). Comparison with the GO loop illustrates the lower saturation and the higher enclosed surface of NO material.

\section{B. Anisotropic Material Model Based on Magnetic Energy Neglecting Hysteretic Behavior}

The material model for the grain oriented material is an anisotropic model based on the magnetic energy that returns a magnetization vector $M$ as a function of an induction vector B. It is a variant to the model described in [11] that uses the field $\mathbf{H}$ as input and is based on coenergy instead of energy. The material model represents the function $\mathbf{M}=\mathbf{M}(\mathbf{B})$ that is used in the FEM-see (2) - to calculate the field patterns. The iron losses are computed a posteriori.

Complementary to [11], we start from the magnetic energy:

$$
W_{\mathrm{em}}(\mathbf{B})=\int_{\mathbf{0}}^{\mathbf{B}} \mathbf{H}(\mathbf{B}) \cdot \mathrm{d} \mathbf{B} .
$$




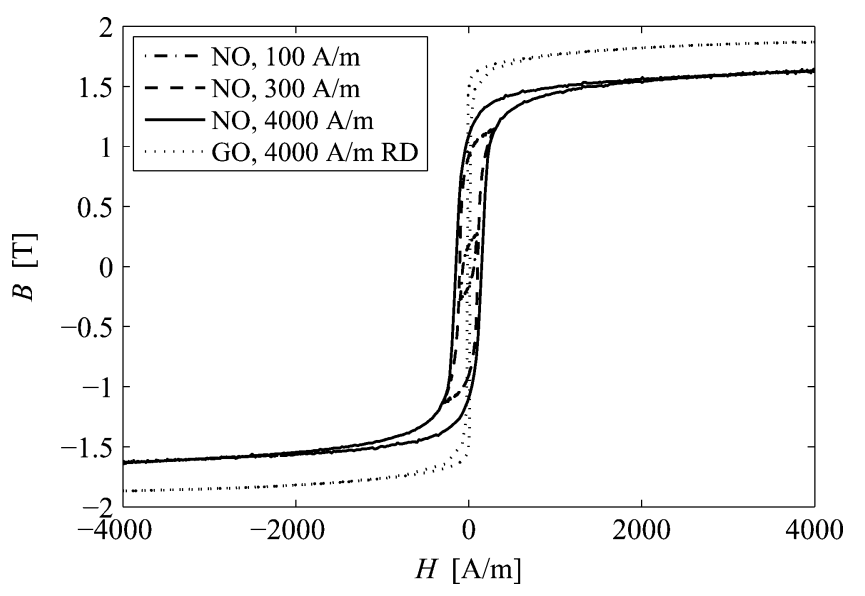

Fig. 8. Hysteresis loops for NO material M700-50 and GO loop in rolling direction as reference for comparison.

The magnetic field can be calculated by taking the gradient from the energy function:

$$
\mathbf{H}(\mathbf{B})=\nabla_{B} W_{\mathrm{em}}(\mathbf{B})=\frac{\partial W_{\mathrm{em}}(\mathbf{B})}{\partial B_{x}} \mathbf{1}_{x}+\frac{\partial W_{\mathrm{em}}(\mathbf{B})}{\partial B_{y}} \mathbf{1}_{y}
$$

This expression can be used only in absence of losses. The required magnetization vector can be found from:

$$
\mathbf{M}(\mathbf{B})=\nu_{0} \mathbf{B}-\mathbf{H}(\mathbf{B}) .
$$

Notice that vectors $\mathbf{M}, \mathbf{B}$, and $\mathbf{H}$ do not necessarily have the same direction.

The numerical construction of the energy function $W_{\mathrm{em}}(\mathbf{B})$, shown in Fig. 9 for GO material, starts from single valued magnetization characteristics along the different directions. A single valued $B H$-characteristic is made for each direction by using the peak values of the experimentally determined hysteresis loops. Then, the energy is calculated using (3). In the $\left(B_{x}, B_{y}\right)$ plane, quasi-static measurements along a direction with angle $\alpha$ give rise to points on a line through the origin with angle $\alpha$ : a function $W_{\mathrm{em}}=W_{\mathrm{em}}\left(B_{\alpha} \cos \alpha \mathbf{1}_{x}+B_{\alpha} \sin \alpha \mathbf{1}_{y}\right)=W_{\mathrm{em}}\left(B_{\alpha}\right)$. All available points in the energy map are on radial lines shown in Fig. 6, with a higher resolution near the origin than in the region for high $B_{x}$ and $B_{y}$. By interpolation, the energy map was reconstructed in an equidistant rectangular grid of points.

\section{Iron Loss Model (Loss Separation)}

For the iron loss evaluation in the electrical machine, the statistical loss model [13], [12] was applied.

From the simulated field waveforms in the machine, we observe that no minor loops are present (Section IV). Consequently, to find the losses, the considered loss model must deal with arbitrary flux waveforms, but not with minor loops. Moreover, we observe that the volume of the soft magnetic material in which nonunidirectional field patterns are present, is limited. We neglect the influence of elliptical or rotational effects on the electromagnetic losses in the material.

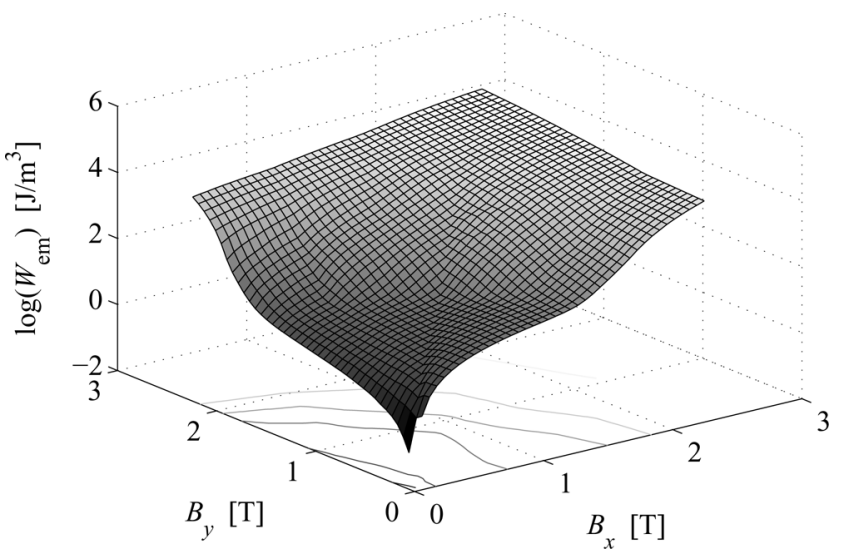

Fig. 9. Logarithm of the energy function $W_{\mathrm{em}}\left(B_{x}, B_{y}\right)$.

1) Hysteresis Loss Component: As minor loops do not occur, the hysteresis loss is independent of the waveform. It is determined by the peak value of the induction. For a fast evaluation of the loss model, a 2-D lookup table was made that contains the hysteresis loss for several values of the peak value vector $\mathbf{B}_{\mathrm{p}}$ lying in the $\left(B_{x}, B_{y}\right)$ plane.

2) Classical Loss Component: The energy loss per cycle of the lamination with thickness $d$ and conductivity $\sigma$ depends on the time derivative of $B(t)$ :

$$
W_{\mathrm{cl}}=\frac{1}{12} \sigma d^{2} \int_{0}^{T}\left(\frac{\mathrm{d} B}{\mathrm{~d} t}\right)^{2} \mathrm{~d} t .
$$

The conductivity was measured based on the 4-point method: $\sigma=(l / S)(I / V)$ where $l$ and $S$ are the length and cross-section of the strip, $I$ is the imposed dc current and $V$ is the measured voltage. For GO, $\sigma$ is $2.0 \mathrm{MS} / \mathrm{m}$, equal in $\mathrm{RD}$ and TD direction. For the NO material, $\sigma$ is $3.2 \mathrm{MS} / \mathrm{m}$.

3) Excess Loss Component: An expression for the instantaneous excess loss is given in [12]:

$$
P_{\text {exc }}=\frac{n_{0} V_{0}}{2}\left(\sqrt{1+\frac{4 \sigma G S}{n_{0}^{2} V_{0}}\left|\frac{\mathrm{d} B}{\mathrm{~d} t}\right|}-1\right)\left|\frac{\mathrm{d} B}{\mathrm{~d} t}\right| .
$$

Here, $n_{0}$ and $V_{0}$ are functions of $B_{\mathrm{p}}$ and should be fitted from measured loops at several frequencies and amplitudes; $n_{0}$ is the number of simultaneously active magnetic objects for frequency $f \rightarrow 0$ and $V_{0}$ defines the statistics of the magnetic objects. The dimensionless coefficient $G=0.1356$ and the lamination cross section $S$ are known constants.

The fitting of $n_{0}$ and $V_{0}$ is done separately for many $B_{\mathrm{p}}$ values and for each considered field orientation. From [13, p. 424-425], it is shown that $n$ depends on the excess field $H_{\text {exc }}$, expecting, as a first approximation, a linear dependence:

$$
n\left(H_{\text {exc }}\right)=n_{0}+\frac{H_{\text {exc }}}{V_{0}} .
$$

Here, $n_{0}$ and $V_{0}$ can be fitted starting from the loss of a measured loop $P_{\text {meas }}$ that is obtained for several $B_{\mathrm{p}}$ and frequencies. Given $P_{\text {meas }}$, the hysteresis loss and the classical loss computed as explained above, $H_{\text {exc }}$ can be found from

$$
H_{\text {exc }}=\frac{P_{\text {meas }}-P_{\mathrm{h}}-P_{\mathrm{cl}}}{4 B_{\mathrm{p}} f}
$$




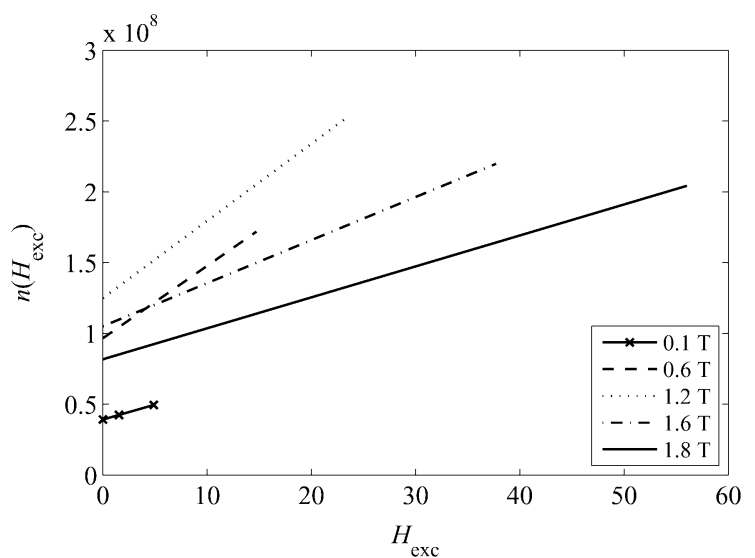

Fig. 10. For GO in the RD, $n$ as a function of $H_{\text {exc }}$.
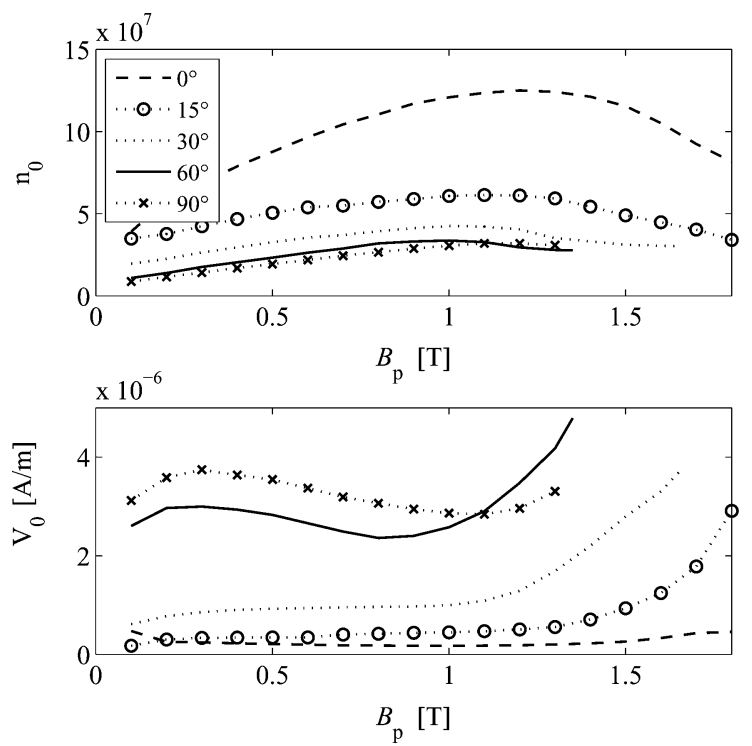

Fig. 11. For GO (a) active regions $n$ as a function of the frequency; (b) and (c): functions $n_{0}\left(B_{p}\right)$ and $V_{0}\left(B_{p}\right)$ in the rolling direction.

wherein $P_{\mathrm{h}}=f W_{\mathrm{h}}$ is the hysteresis loss in $\mathrm{W} / \mathrm{m}^{3}$, and the classical loss is obtained by multiplying (6) by $f$ or for sinusoidal fields, as $P_{\mathrm{cl}}=\left(\pi^{2}\right) /(6) \sigma d^{2} B_{\mathrm{p}}^{2} f^{2}$.

With this $H_{\text {exc }}$, the number of active magnetic objects is

$$
n\left(H_{\mathrm{exc}}\right)=\frac{4 \sigma G S B_{\mathrm{p}} f}{H_{\mathrm{exc}}} .
$$

With (9) and (10), the curves (8) for every $B_{\mathrm{p}}$ can be fitted, as shown in Fig. 11 for GO material. The parameter $S$ was chosen equal to $1 / G$. For all considered directions, the function $n\left(H_{\text {exc }}\right)$ is approximated by a straight line. This is an acceptable approximation. For the NO material, similar curves for $n_{0}$ and $V_{0}$ were determined.

The model is unidirectional, while the field and induction have an elliptical locus in some parts of the geometry. Fig. 12 shows the loci in several points of the geometry. It is however observed that in the majority of the surface, the field in the dominant direction is much larger than the field along the small axis of the ellipse. Therefore, we evaluated the losses as unidirectional losses along the dominant direction of the field in a point.
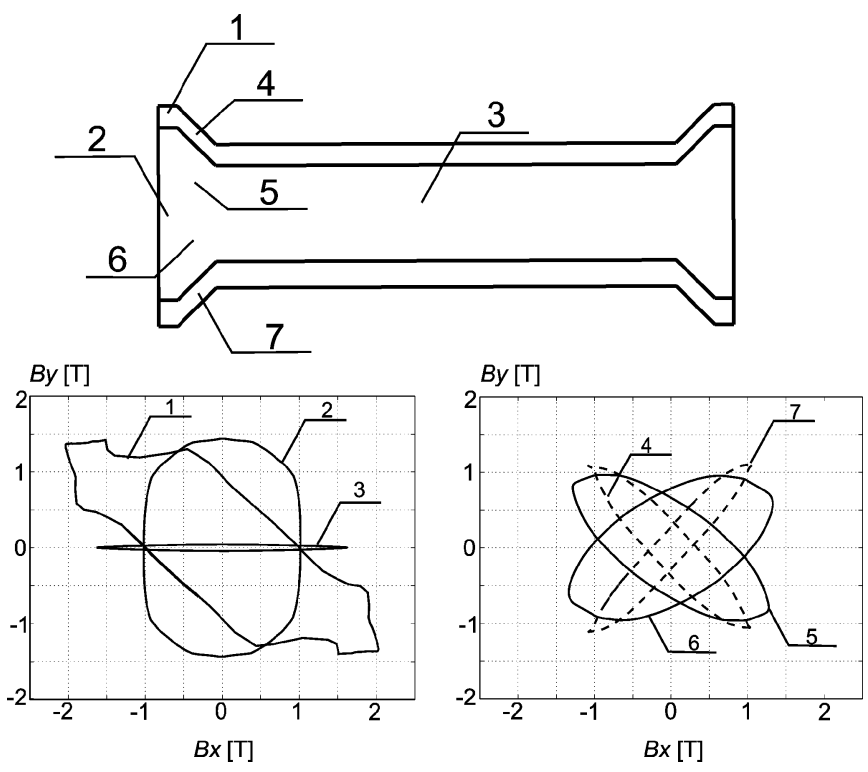

Fig. 12. Loci in the $\left(B_{x}, B_{y}\right)$ plane at several positions in the stator tooth.

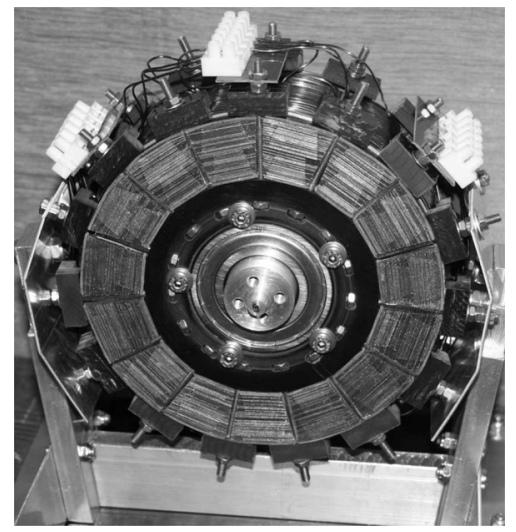

Fig. 13. The stator with 15 teeth can be seen after removal of the rotor.

Both for the direction and the amplitude, an interpolation was made for the functions $n_{0}$ and $V_{0}$.

\section{Simulations AND EXPERIMENTS}

\section{A. Experimental Setup}

The stator of the AFPMSM can be seen in Fig. 13. It is connected to a 2-pole induction machine of $7.5 \mathrm{~kW}$ via a torque sensor. All phase voltages consist of five concentrated windings (five teeth) in series, but the machine was made in such a way that the voltage of each of the 15 windings is accessible separately. The voltage was measured by a National Instruments data-acquisition system with sampling speed up to $250 \mathrm{kSam}-$ ples per second. The speed is obtained from an optical position sensor. The induction machine is supplied by an $11 \mathrm{~kW}$ inverter that is controlled by LabVIEW. The setup was constructed to measure the EMF and to do the run-out test.

\section{B. Field Waveforms in the GO and NO Material}

The magnetic field waveforms obtained from 2-D FEM during the passing of the magnets is shown in Fig. 14. The 


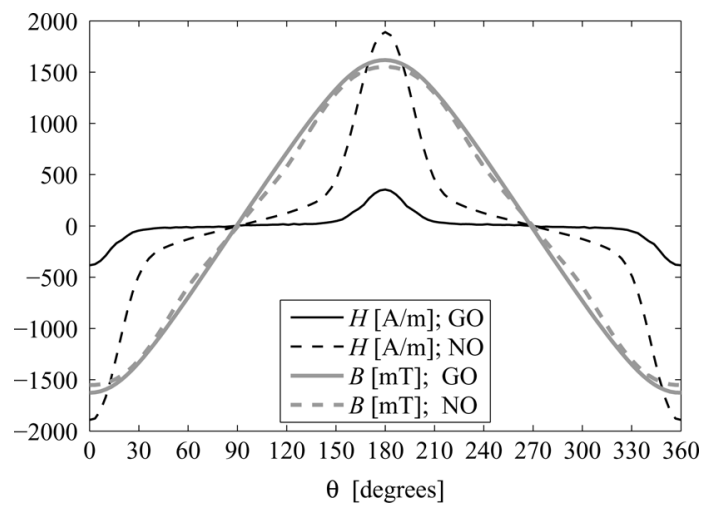

Fig. 14. Waveforms of magnetic field and induction in the center of the stator yoke at no-load for the NO and GO material.

(a)
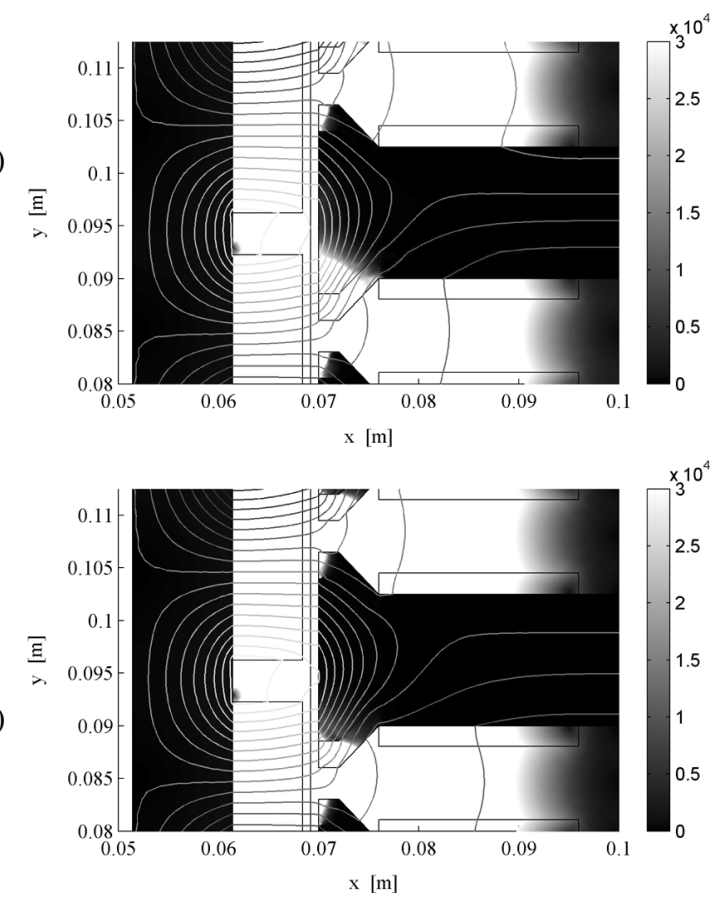

Fig. 15. Field pattern in layer 2 with 5.8 A stator current for (a) GO material and (b) NO material. The scale is in $\mathrm{A} / \mathrm{m}$.

difference between GO and NO material depends on the stator current: at no-load, the induction waveform is almost the same for both materials (peak of 1.6 T), but the corresponding field is about $2000 \mathrm{~A} / \mathrm{m}$ in the NO material, and approximately ten times less in the GO material. When load current is added, the induction of the NO material remains almost the same, because it is saturated; the GO material however reaches a much higher induction, giving rise to higher torque.

The difference in field pattern in a NO and a GO stator tooth can be seen in Fig. 15. If the stator contains GO material, the field lines have tendency to align with the RD.

\section{Torque and EMF Comparison Between $\mathrm{GO}$ and NO}

At no load, Fig. 16 shows the EMF of a tooth $E_{\mathrm{t}}$ per 1000 rpm. The total EMF of a phase $E_{\mathrm{ph}}$ consists of five teeth in series, whose EMFs are shifted in phase. The total EMF per phase can be found as

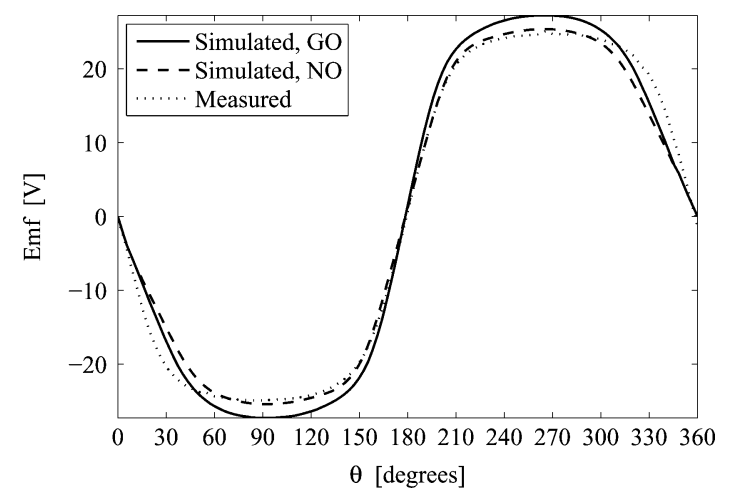

Fig. 16. Electromotive force at $1000 \mathrm{rpm}$ in a tooth winding at no load, simulated for NO and GO material, and measured. The abscis is the electrical rotor angle, which is 8 times the mechanical angle.

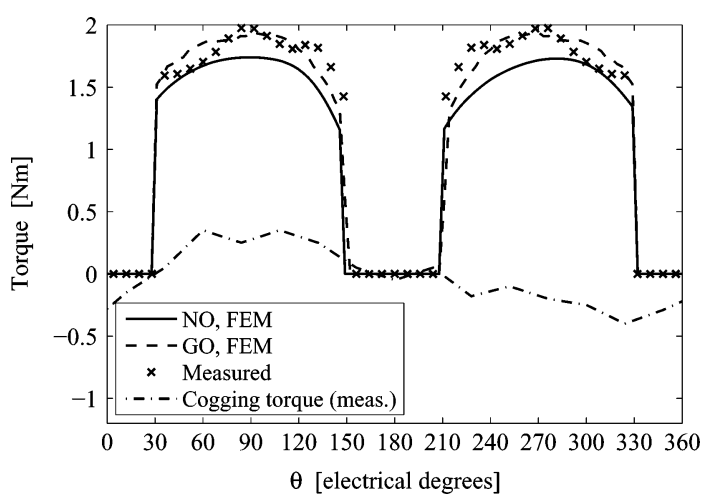

Fig. 17. Torque of one tooth as a function of the electrical rotor angle. A current of $5.78 \mathrm{~A}$ was injected between $30^{\circ}$ and $150^{\circ}$, and between $210^{\circ}$ and $330^{\circ}$. The cogging torque was removed both in measurements and simulations.

$$
E_{\mathrm{ph}}=E_{\mathrm{t}}+2 \cos \left(12^{\circ}\right) E_{\mathrm{t}}+2 \cos \left(24^{\circ}\right) E_{\mathrm{t}}=4.7834 E_{\mathrm{t}} .
$$

The EMFs in the three phases are shifted $120^{\circ}$ so that-when connecting them in star-the no-load output voltage is $4.7834 \sqrt{3} E_{\mathrm{t}}$. It is observed that the GO material produces almost the same EMF as the NO at no-load. The difference between the simulated EMF for GO material and the measured $\mathrm{EMF}$, is within the tolerance of the measurements and the numerical accuracy of the simulations.

The torque at large load, shown in Fig. 17, illustrates that the GO material has about $10 \%$ more torque for the same current, which was the nominal current of $5.78 \mathrm{~A}$. The ripples on the torque profile are caused by convergence problems in one or more of the six layers of the FEM. The measured torque profile corresponds well with the simulations for GO material.

\section{Loss Comparison Between $\mathrm{GO}$ and $\mathrm{NO}$}

Fig. 18 makes a comparison of iron losses in all 15 stator teeth for several rotor speeds, at no-load. The different components, i.e., classical, hysteresis and excess loss in the stator teeth, see e.g., (6) and (7) are shown. It is seen that NO material has about seven times more losses than GO for the complete speed range. For a machine of more than $3 \mathrm{~kW}$ nominal power, the $30 \mathrm{~W}$ 
GO

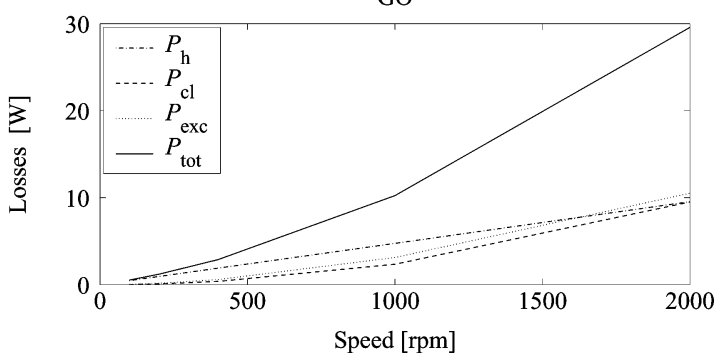

$\mathrm{NO}$

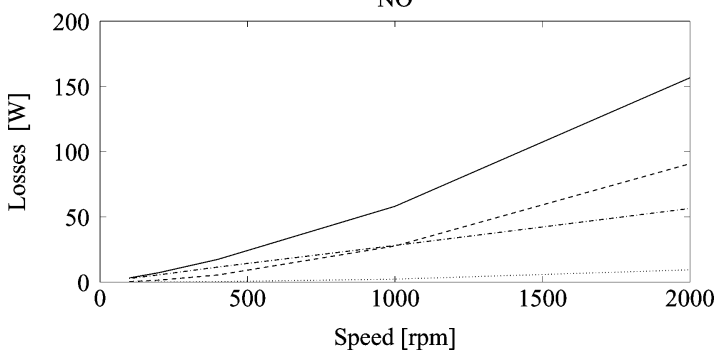

Fig. 18. Stator iron losses for the whole machine as a function of the speed, for GO and NO material.

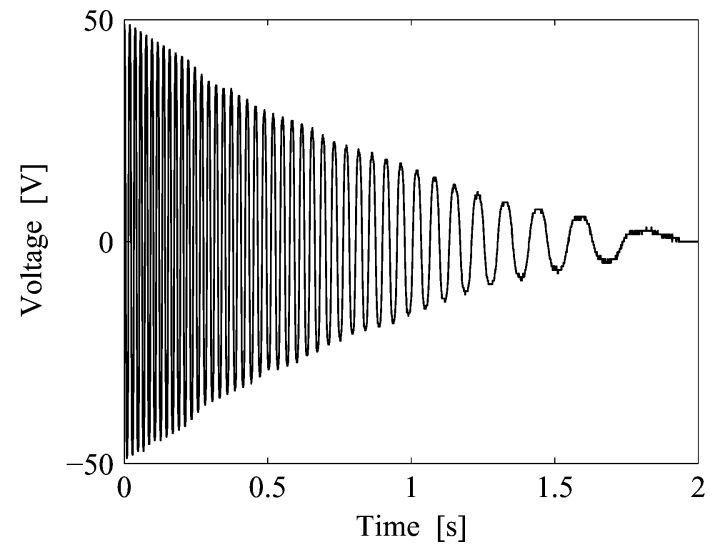

Fig. 19. Run-out test of the machine at no-load starting from $400 \mathrm{rpm}$.

losses for GO material are low, thanks to the low losses per $\mathrm{kg}$ of the material and to the low mass of iron in the machine.

The losses at no-load can be compared with the losses obtained from a run-out test: $J(d \Omega) /(d t)=-T_{\text {loss }}$, with $\Omega$ the mechanical speed and $T_{\text {loss }}$ the loss torque. The inertia $J$ was calculated to be $0.0107 \mathrm{~kg} \cdot \mathrm{m}^{2}$. Fig. 19 shows a run-out test from $400 \mathrm{rpm}$. The recorded voltage makes it possible to determine at any moment the speed and its time derivative. From these data, the experimental total loss can be found as a function of the frequency. This total loss consists of losses in bearings, magnetic material in stator and rotor, and magnets. A comparison revealed that the measured total loss (obtained from run-out test) was much higher than the simulated loss corresponding with stator iron loss only (Fig. 18): at $400 \mathrm{rpm}$, it was $15.0 \mathrm{~W}$ compared to $2.89 \mathrm{~W}$ iron loss in the GO stator teeth. It is assumed that the losses in the bearings are dominant.

\section{CONCLUSION}

For an AFPMSM whose stator flux is flowing in axial direction in the major part of the stator, GO material was compared with NO material. With GO material, the machine has about 7 times less iron loss at the same speed, and a $10 \%$ higher torque for the same current. Nevertheless, the EMF at no-load is almost the same for both materials. For a given torque, the GO material causes a $10 \%$ higher torque-to-current ratio which makes it possible to reduce the copper losses-quadratic with the current-by about $20 \%$. Alternatively, because of the lower iron losses, it is possible with GO material to allow larger copper losses without increasing the temperature of the machine. This means a higher stator current and more torque. We conclude that for the considered type of PMSM, it is worth the extra cost to use GO material.

\section{ACKNOWLEDGMENT}

This work was supported by the FWO project G.0082.06, by the GOA project BOF 07/GOA/006, and the IAP project $\mathrm{P} 6 / 21$. The first author is a postdoctoral researcher for the "Fund of Scientific Research Flanders" (FWO).

\section{REFERENCES}

[1] A. Cavagnino, M. Lazzari, F. Profumo, and A. Tenconi, "A comparison between the axial flux and the radial flux structures for PM synchronous motors," IEEE Trans. Ind. Appl., vol. 38, no. 6, pp. 1517-1524, 2002.

[2] T. F. Chan and L. L. Lai, "An axial-flux permanent-magnet synchronous generator for a direct-coupled wind-turbine system," IEEE Trans. Energy Convers., vol. 22, no. 1, pp. 86-94, 2007.

[3] S. Brisset, D. Vizireanu, and P. Brochet, "Design and optimization of a nine-phase axial-flux PM synchronous generator with concentrated winding for direct-drive wind turbine," IEEE Trans. Ind. Appl., vol. 44, no. 3, pp. 707-715, 2008.

[4] A. B. Letelier, D. A. Gonzalez, J. A. Tapia, R. Wallace, and M. A. Valenzuela, "Cogging torque reduction in an axial flux PM machine via stator slot displacement and skewing," IEEE Trans. Ind. Appl., vol. 43, no. 3, pp. 685-693, 2007.

[5] G. S. Liew et al., "Analysis and performance evaluation of an axial-field brushless PM machine utilising soft magnetic composites," in Proc. IEEE Int. Electric Machines and Drives Conf. (IEMDC 2007), Antalya, Turkey, May 3-5, 2007, pp. 153-158.

[6] A. Di Gerlando, G. M. Foglia, R. Perini, and M. Ubaldini, "Permanent magnet synchronous machines with concentrated coil armature windings: Analysis and test validation of single stator-double rotor, axial flux machines," Elect. Eng., vol. 90, pp. 65-77, 2007.

[7] T. F. Chan, L. L. Lai, and S. Xie, "Field computation for an axial flux permanent-magnet synchronous generator," IEEE Trans. Energy Convers., vol. 24, no. 1, pp. 1-11, 2009.

[8] P. Virtic, P. Pisek, M. Hadziselimovic, T. Marcic, and B. Stumberger, "Torque analysis of an axial flux permanent magnet synchronous machine by using analytical magnetic field calculation," IEEE Trans. Magn., vol. 45, no. 3, pp. 1036-1039, Mar. 2009.

[9] A. Parviainen, M. Niemelä, and J. Pyrhönen, "Modeling of axial flux permanent-magnet machines," IEEE Trans. Ind. Appl., vol. 40, no. 5 , pp. 1333-1340, 2004.

[10] F. Fiorillo, L. Dupré, C. Appino, and A. M. Rietto, "Comprehensive model of magnetization curve, hystersis loops, and losses in any direction in grain-oriented Fe-Si," IEEE Trans. Magn., vol. 38, no. 3, pp. 1467-1476, May 2002.

[11] T. Pera, F. Ossart, and T. Waeckerle, "Field computation in nonlinear anisotropic sheets using the coenergy model," IEEE Trans. Magn., vol. 29, no. 6, pp. 2425-2427, Nov. 1993.

[12] E. Barbisio, F. Fiorillo, and C. Ragusa, "Predicting loss in magnetic steels under arbitrary induction waveform and with minor hysteresis loops," IEEE Trans. Magn., vol. 40, no. 4, pp. 1810-1819, Jul. 2004.

[13] G. Bertotti, Hysteresis in Magnetism. San Diego, CA: Academic, 1998. 